Catherine FRIER

Marie-Cécile GUERNIER

LIDILEM Université Stendhal Grenoble 3

\title{
Paroles de lecteurs : et si les usages scolaires empêchaient de pratiquer la lecture?
}

(Article paru dans Repères n 35, 2007)

Ce texte n'est que l'amorce d'une analyse portant sur un recueil de données réalisé au cours de l'année 2005- 2006, dans le cadre d'une enquête sur le rapport aux écrits disciplinaires des 15- 18 ans. Ce recueil de données à pris plusieurs formes : observations comparatives de pratiques de lecture et d'écriture dans les différentes disciplines au collège et au lycée, enregistrements vidéo de séances, entretiens d'élèves et d'enseignants, questionnaires, recueil de productions d'élèves. Nous avons enquêté dans deux établissements de la région grenobloise: le CLEPT $^{1}$, où notre attention s'est portée tout particulièrement sur les élèves de première, et le collège Louis Mauberet de la Mure où nous avons enquêté auprès des élèves de $3^{\mathrm{e}}$ générale, $3^{\mathrm{e}}$ d'insertion et $3^{\mathrm{e}}$ segpa. L'un des volets de la recherche portant plus particulièrement sur le rapport à la lecture des élèves en fin de cycle de collège, nous avons naturellement été concernées par la problématique relative aux «ratés de l'apprentissage de la lecture ».

La question de l'évaluation de la maîtrise de la lecture est complexe. Il est à peu près acquis aujourd'hui que les réponses relèvent de paradigmes divers : cognition, représentation sociale et culturelle, psychologie et psychanalyse, acculturation, didactique. Cependant une chose est sûre : pour mieux comprendre comment la compétence lecturale se construit - il est en effet indéniable que même le lecteur qualifié de faible a construit une compétence dans le domaine de l'écrit - le chercheur doit s'intéresser au sujet lecteur lui-même, à son activité et à ce qu'il y investit intellectuellement, psychiquement, socialement et culturellement. Dans cette perspective, nous sommes loin d'avoir investi toutes les pistes.

La didactique de la lecture telle que nous la concevons est une didactique centrée sur le sujet, et qui prend en compte son rapport à l'écrit (Barré de Miniac, 2000). La conquête de la langue écrite est un acte impliqué, relevant d'une appropriation individuelle, et c'est pourquoi nous pensons que seul ce « parti pris de l'apprenant » (Delamotte et Alii, 2000), peut favoriser la réussite des apprentissages dans ce domaine.

Cette étude, en cours de réalisation, devrait permettre d'identifier les éléments concernant l'apprentissage de l'écriture et de la lecture qui, dans le parcours scolaire de ces élèves, ont pu constituer à un moment donné des obstacles. Elle devrait également nous aider à repérer à quels moments spécifiques du parcours ces obstacles se sont révélés, et dans le cadre de quelles modalités d'apprentissage de l'écrit (école maternelle et découverte du code, CP et apprentissage de la lecture, cycle 2 et 3, collège et approche littéraire, lycée et exercices formels).

L'article, tout en rendant compte des réponses des élèves, présentera et analysera leur profil de lecteur ainsi que leur rapport à la lecture. Cette analyse devrait nous permettre de mettre en évidence les éléments liés à l'écrit en milieu scolaire et perçus comme sources de ratés de l'apprentissage et causes de l'échec.

\footnotetext{
${ }^{1}$ Collège Lycée Elitaire pour tous, établissement qui accueille des collégiens et lycéens « raccrocheurs ».
} 


\section{Lire au collège : ils savent faire, mais n'aiment pas ça...}

Dans cette partie du texte, nous allons nous centrer sur une catégorie d'élèves qui a retenu notre attention, mais dont on parle finalement assez peu : il ne s'agit ni des $4 \%$ d'élèves en grande difficulté de lecture à la fin de la scolarité élémentaire, suspectés d'illettrisme ou de dyslexie, ni des élèves très bons lecteurs à leur entrée en $6^{\mathrm{e}}$, (capables d'interpréter des textes de nature différente, de relier les informations entre elles, de comprendre les informations implicites du texte, etc.), mais plutôt d' une catégorie intermédiaire, hétéroclite, car difficile à cerner de manière unilatérale, et somme toute assez banale, puisque majoritaire, d'élèves sachant lire mais déclarant ne pas aimer pas ça, adolescents pour qui la lecture est d'abord perçue comme une activité scolaire, souvent désincarnée, en tous cas peu incarnée dans des pratiques sociales et " familiales », susceptibles de donner vie à un véritable « habitus » lectural :

«En effet, arrivés à l'âge de la quatrième, il ne reste que fort peu d'enfants qui se définissent comme des lecteurs, qui déclarent aimer lire(...). L'inégalité de sollicitation autour de la lecture que l'on constate dès la petite enfance, se retrouve au collège, si elle n'a pas été compensée. On retrouve le même problème de familiarisation avec l'écrit constaté à l'entrée du CP. Ainsi, pour beaucoup d'entre eux, et même chez ceux qui n'ont pas de problèmes majeurs de scolarité, lire et écrire demeurent des activités purement scolaires, dans la mesure où ces pratiques ne s'intègrent pas dans des habitudes familiales, s’effectuent peu et rarement de façon «naturelle», spontanée ». (Chartier, 2006 : 208)

Ces élèves ne sont pas à proprement parler de «mauvais lecteurs », ce sont plutôt des lecteurs fragiles, inaboutis, ou « au bord de la lecture ».

Ces premiers constats nous amènent à considérer la lecture comme une compétence inachevée au collège : si les élèves manifestent un certain nombre de savoir- faire (lire à voix haute de façon relativement fluide, être capables de répondre à des questions portant sur un texte), l'apprentissage est loin d'être entièrement terminé, et beaucoup de choses restent encore à construire dans ce domaine.

A partir de ces constats nous avons eu envie d'interroger la question de l'apprentissage continué de la lecture au collège : apprend-on encore à lire au collège ? Quoi et de quelle façon ? Doit-on considérer que la scolarité élémentaire règle tous les problèmes relatifs à la compréhension ? A la médiation au livre ? Les élèves sont ils suffisamment outillés affronter les textes, notamment littéraires, qui leur seront proposés au collège ? Et qu'en disent-ils ? Dans quelle mesure les pratiques pédagogiques mises en œuvre peuvent-elles favoriser ou au contraire gêner la continuation des apprentissages dans ce domaine ? Et quels en sont les objectifs explicites ? Implicites?

L'objectif ici est donc de décrire et d'analyser le rapport à la lecture de ces élèves, de mieux comprendre un certain nombre de leurs difficultés, et de scruter leur manque d’appétence vis-à-vis de la lecture.

Pour répondre à ces questions, nous avons fait le choix, une fois n'est pas coutume, de donner la parole aux élèves, en leur faisant exprimer leur du point de vue. Nous avons réalisé 10 entretiens semi-directifs auprès d'élèves de troisième ordinaire du collège Louis Mauberet à la Mure. Précisons seulement que ces élèves étaient volontaires, mais que sur les 10, 6 ont déclaré lors de la présentation préalable de l'enquête à la classe, ne pas aimer lire et avoir envie d'en parler. Nous avons trouvé cette démarche intéressante, compte tenu du poids symbolique et des représentations sociales de la lecture à l'école, et c'est principalement sur l'analyse de ces 6 entretiens que sera construit notre analyse. L'entretien recoupait en grande partie les rubriques utilisées auprès des élèves du Clept, mais était beaucoup plus orienté sur le rapport à la lecture (pratique et représentations) scolaire/périscolaire de l'élève, la perception de ses compétences, de ses difficultés, de ces réticences, la place enfin attribuée à la lecture dans le parcours scolaire. Les entretiens ont été réalisés au collège, à la fin de l'année scolaire (au moment des conseils), et ce détail n'est pas anodin : ces élèves quittant le collège l'an prochain, ils ont pu exprimer les choses avec une certaine distance, à la manière d'un bilan à mi -parcours du cycle secondaire, bien entendu sans souci d'évaluation institutionnelle de leur propos. Nous aurions pu présenter nos résultats sous forme de petites monographies, tant chaque individu entretient ave cette activité une relation intime, unique, irréductible à des généralités. Néanmoins, dans un souci d'économie, nous avons préféré dans un premier temps présenter les thématiques qui émergent à la toute première lecture de ces entretiens. Il n'empêche que le lecteur pourra identifier, au gré des citations, dans les propos parfois contradictoires de ces élèves, la complexité de ce rapport individuel à la lecture.

Que disent-ils de la lecture, et comment interpréter leurs propos?

Voici les grands axes de notre analyse

\section{La lecture et moi}

Toute une série de questions de l'entretien visait à amener les élèves d'une part à donner leur point de vue sur l'activité lecture en général, d'autre part à s'évaluer en tant que lecteur. Ils pouvaient s'exprimer " librement ", étant entendu que leurs propos n'auraient aucune incidence ni sur leurs résultats scolaires ni sur leur image d'élève. Les propos recueillis font ressortir des réactions épidermiques : fortement modalisés, utilisant le lexique des sentiments, ils témoignent en effet de cette volonté de dire avec un certain excès, le rejet « en bloc » de l’activité lecture : 
«La lecture, j'aime pas du tout, c'est même une maladie » (Michaël)

«j' ai horreur de lire, j'ai jamais aimé ça » (Naïm)

«ça m'énerve, je suis pas un passionné » (Cyrille)

«ça m'ennuie de lire » (Antoine)

" j'ai horreur de ça » (Cyrille)

Mais si l'on cherche à dépasser ce premier stade, à creuser ce «cri du cœur », on obtient des choses beaucoup plus nuancées, moins tranchées. Lorsqu'on leur demande par exemple d'évaluer leur compétence en lecture, si certains avouent connaître quelques difficultés, évoquant par exemple le manque de rapidité :

«j'arrive pas trop à lire aussi, alors euh je vais pas, je lis plus lentement que les autres, même dans ma tête, des fois y pose des questions j'ai pas fini de lire donc » (Elisa)

« Je lis pas très très bien, je dois lire beaucoup moins vite que quelqu'un qui lit plus souvent que moi » (Michaël),

la plupart considèrent qu'ils savent lire :

« Je sais lire, je lis bien mais j’aime pas », «j’ai pas de dyslexie hein » (Benoît)

«Depuis que je suis tout petit je sais lire» (Cyrille)

«Mais ça me dérange pas de lire un texte d'une page si la prof elle me demande y pas de problème » (Michaël).

Le fait de ne pas aimer lire n’est donc pas spontanément associé à des problèmes de lecture (décodage ou compréhension), mais plutôt à manque d'habitude, de pratique :

«maintenant je sais pas si c'est une éducation ou si c'est naturel euh le fait de pas pouvoir lire parce que moi mes parents enfin ma mère elle lit un petit peu enfin rien de bien extraordinaire, mais euh mon père lui il lit pas donc y a pas trop de lecture dans la famille, donc je me demande si ça vient de ça, si du fait que j'ai pas été trop, enfin si c'est le fait qu je suis pas très destiné à lire »( Michaël), "ben mes sœurs elles lisent beaucoup, mais moi non, peut-être parce que elles elles ont lu beaucoup, ben en fait eux ils lisent par plaisir, ils ont été habitués à lire quoi, et moi ben j'ai jamais été habitué à lire » (Laurent), «ben par rapport à d'autres que je vois et qui sont toujours avec leur bouquins, moi je lis presque pas» (Roselyne).

Le message est donc le suivant : en gros je sais lire, le problème ne se situe pas là, et pourtant je ne lis pas, d'abord parce que, contrairement à d'autres, je n'en ai jamais pris l'habitude.

Comment ces élèves se représentent-ils le « bon lecteur » ?

Pour nos témoins, celui qui lit bien, sait lire à voix haute : «quelqu’un qui lit normalement, qui bute pas sur les mots tout le temps, qu'on arrive à comprendre quand y lit un texte quoi, pas qui s'arrête, qui laisse un blanc quand il lit un texte » (Elisa), « quelqu’un qui hésite pas » (Antoine), « ben déjà quelqu’un qui bégaie pas, qui sait ce qu’il dit » (Cyrille).

De ce point de vue là, mise à part Elisa, tous se considèrent comme performants.

C’est aussi « quelqu'un qui lit vite » (Cyrille, Antoine), « en comprenant l'histoire » (Antoine). Or certains avouent connaître quelques difficultés à ce niveau là : «J'arrive bien à lire, mais je comprends pas toute l'histoire » (Antoine).

C'est enfin et surtout quelqu'un qui aime lire et qui le fait sans être contraint : «quelqu'un qui sait se mettre dans le livre quoi, pas lire une page ou deux fermer le livre, cinq minutes après revenir, relire une page ou deux, pas lire parce qu'on doit lire, lire parce qu'on aime lire » (Cyrille), " aimer lire déjà, pas être contraint, pas se dire chaque soir comme ça je dois lire ça, aimer l'histoire quoi, lire ce qui nous plaît, et comprendre ce que l'auteur veut dire » (Yohan).

C’est pourquoi dans l'ensemble nos témoins ne se considèrent pas comme de bons lecteurs : s’ils savent lire, ils ne comprennent pas toujours ce qu'ils lisent, et le font souvent sans plaisir.

\section{La lecture littéraire : point névralgique des tensions}

Mais de quelle lecture est-il question en fait ? Très vite l'enquêteur réalise que pour ses élèves, lire signifie « lire au collège » et en particulier « en français ». Le type de lecture qui cristallise le rejet évoqué plus haut est représenté par le texte littéraire lu et étudié en classe de français : « Mais un livre comme ça non, un roman ça j’aime pas du tout, les récits, les romans, les livres qu'on nous distribue au collège quoi » (Antoine)

«Les livres qu'on lit en français, Anne Franck, je vois pas à quoi ça va me servir » (Naïm) 
«mais je lis pas des livres, des romans, je lis pas un livre pour moi, prendre un livre à la bibliothèque, non, j'ai pas envie », «j'ai même pas fini de le lire (le journal d'Anne Franck), quand j'aime pas, ça m'intéresse pas alors j'ai pas envie de lire » (Elisa)

«Enfin la lecture ça doit être normalement un plaisir, mais moi j’arrive à lire des livres entiers mais on se fait pas plaisir dessus, on retient pas » (Antoine)

Donc la lecture qui est en cause c’est la lecture littéraire, celle pratiquée dans les autres disciplines semblant poser beaucoup moins de problèmes :

- $\quad$ " un long texte de SVT on y arrive toujours (...) parce que j'ai l'impression qu'en SVT c'est des choses nouvelles et qu'on nous explique tout de suite, alors qu'en français la lecture on dit que c'est su donc... », " en français c'est des choses qu'on apprend depuis le début de notre vie quoi, alors qu'en SVT y a beaucoup de choses qu'on connaît pas », (Dimitri)

- $\quad$ "la plupart de temps, c'est sur les textes, alors y a pas grand-chose à apprendre, c'est toujours la même chose sur les textes » (Antoine)

- ça me dérange pas de lire mon livre d'histoire par exemple ou de maths ou de physique » (Cyrille)

Soulignons au passage car cela n'est pas anodin, que pour ces élèves, et nous avons retrouvé le même discours chez les élèves plus âgés du CLEPT, la discipline français apparaît comme un objet d'apprentissage non identifié, une matière aux contours un peu obscures où l'on ressasse à l'infini des "choses ", "des trucs » sans mobile apparent: "c'est juste des textes à lire, et après un truc, un contrôle » (Benoît), " des études de documents pour arriver à un but, pour arriver à quelque chose quoi, un point de grammaire ou un truc comme ça » (Dimitri), et dont ils ont du mal à cerner les enjeux.

Venons-en à présent aux difficultés que les élèves évoquent à propos de la lecture littéraire en français. Autrement dit quels sont les principaux obstacles/ embûches rencontrés par les élèves dans ce domaine ?

Beaucoup évoquent la difficulté à "planter le décor » : "j’arrive pas à imaginer les personnages comment ils sont en réalité, par exemple comme Harry Potter, si on lit qu'il a des cheveux bruns tout ça mais on sait pas comment il est réellement, les paysages j'arrive pas à m'imaginer, j'arrive pas à rentrer dans le livre », (Cyrille), " quand il écrivent il faut vraiment se mettre dans le contexte, etc. et ça j'ai du mal à arriver à me mettre dans le même contexte qu'eux, à savoir dans quel contexte ils étaient quand ils posaient la question » (Michaël), "c'est le début de l'histoire en fait, ça m'intéresse pas ». Ici les élèves semblent souffrir de ce que l'on pourrait appeler un "manque de mise en scène », comme si rien de concret ne les aidait à élaborer mentalement le cadre imaginaire nécessaire à la fiction. S'ils ont du mal à entreprendre ou à poursuivre leur lecture, c'est que rien ne les aide de façon concrète à donner corps à cette fiction, à la rendre palpable, à la relier à des fragments de leur réalité, à lui donner un sens.

Du coup ils évoquent le manque d'intérêt à l'égard de ce type de lecture : "y a des livres je trouve qu'ils sont pas intéressants pour moi » (Cyrille), "j'ai l'impression en fait que je me désintéresse au fur et à mesure que je lis et que je me perds après dans autre chose, alors je lis et des fois je me dis tiens, je sais même plus de quoi elle parle en fait, pourtant je l'ai lu hein, mais y rien qui m'accroche » (Michaël), "c'est le début de l'histoire en fait, ça m'intéresse pas jamais, alors j'abandonne» (Anthony). Ce manque d'intérêt, évoqué de façon récurrente est souvent associé aux thématiques choisies : «j'aime pas les sujets qui mettent dans les livres, j'aime pas ces livres donc », c'est les sujets moi qui me posent problèmes, ces histoire j'aime pas ça, ça m'intéresse pas donc je me donne même pas la peine », "j'aime lire mais pas leur histoires à eux » et à propos du journal d'Anne Franck «y a pas d'action, c'est triste » (Benoît),

Les élèves évoquent également la difficulté à comprendre ce type de textes, qui exigent une lecture analytique, plus experte, vis-à-vis de laquelle beaucoup se sentent démunis : "je me demande si c'est le fait de pas lire assez ou si vraiment j'ai un manque de compréhension avec les écrits euh parce que y a des auteurs mais je comprends pas du tout », " je peux comprendre tout ce qui se dit mais après bon on a vu en cours qu'y avait d'autres trucs qu'ils fallait lire entre les lignes, y a une critique générale, enfin y a une histoire d'origine et y a un truc à côté pour euh, enfin comment dirais-je, faut lire entre les lignes quoi » (Michaël).

Lire en français au collège, c'est aussi être confronté à des textes de plus en plus complexes du point de vue linguistique : «ben le vocabulaire, quand il y a des choses qu'on comprend pas, par exemple là on doit lire Rhinocéros et y a beaucoup de mots que je comprends pas, mais en fait c'est pas tellement le vocabulaire, c'est plutôt la formulation en fait, et puis dans les dialogues on se trompe de personnage » (laurent), un langage inconnu, peu familier, non pratiqué qui entrave la compréhension : « ce qui m'énerve aussi dans les livres qui sont assez anciens, le langage il 
est assez compliqué et puis c'est souvent la même chose, y des phrases faut vraiment calculer chaque mot pur comprendre le sens, sinon deux trois mots à la suite c'est des mots qu'on n'utilise pas maintenant, on comprend pas la phrase donc il faut le relire deux ou trois fois, donc j'aime bien lire un livre sans avoir de coupure » (Cyrille).

David résume assez bien les choses en disant «c'est pas de lire qui me gêne, c'est que la difficulté des livres elle devient compliquée ».

Le cas de Elisa est un peu particulier. Elle est la seule à faire allusion à de réels problèmes de décodage : «j’hésite sur des mots et j'arrive pas à lire un texte sans vraiment m'arrêter ». Au début de l'année, Elisa a demandé à son professeur de français de ne pas lui demander de lire à voix haute devant la classe. Un contrat a été passé : Elisa ne lira que de courts passages, préparés la plupart du temps. Si Elisa ne veut pas lire à voix haute c'est parce qu'elle a peur de se tromper : " j’ai peur de me tromper en fait en lisant, et qu'après on se moque de moi, en fait je relis plusieurs fois dans ma tête pour être sûre que c'est ce mot là ». Son blocage remonte à l'enfance : lorsque on lui demande comment elle a appris à lire, , elle répond : "on apprenait sur des petits mots comme ça, on lisait le mot et on le coupait d'abord", "c'est vers le CM1 qu'on a commencé à se moquer de moi, alors j'osais plus lire et j'avais toujours peur de me tromper et qu'on se moque de moi, c'est pour ça que le lis un peu doucement et je bute sur des mots parce que j'ai peur de me tromper », «dans ma tête je lis bien, c'est les gens en fait qui me gênent ».

Ce qui gêne enfin beaucoup les élèves, c'est la longueur des lectures : " quand on arrive au collège qu'on doit lire des gros livres, ça nous perturbe » (Dimitri), qui de surcroît sont imposées: "quand on nous a imposé ce livre y avait déjà la moitié des élèves qui étaient pas ... enfin ils devaient lire parce qu'il y avait une note à la fin mais autrement » (Dimitri), « j'étais obligé de le lire donc je l'ai lu mais sinon » (Cyrille), « je le fait par obligation, je le fait parce que je devais le faire et que je l'aurais pas fait de mon plein gré » (Michaël), « je me force à les lire » (Naïm).

Enfin ils évoquent la difficulté à lire seul ce type de texte qui nécessite une médiation: "c'est chez moi que j’aime pas » (Benoît), "je les apprécie plus que si je devais lire les bouquins à la maison parce qu'on est tous ensemble, c'est toujours moins long en plus », " on doit les lire tous seuls » (Anthony), et le manque de médiation : "c'est juste avec un livre épais, prenez des notes pour vous y retrouver, mais sans plus quoi » (Dimitri)

En définitive, les propos des témoins donnent l’impression que la lecture littéraire est perçue comme une activité livrée sans le mode d'emploi ni le service après-vente, un objet d'apprentissage non ancré dans un corps de pratiques susceptibles de les relier aux autres pratiques langagières dans la classe. Elle est présentée comme occupant un espace à part, coupé des autres lectures, une zone d'abstraction, faisant principalement appel à une approche intellectuelle, abstraite. Lorsqu'on leur demande ce qu'ils font en français les élèves répondent « on lit des textes ». Le problème pour eux, c'est que les enseignants considèrent qu'ils savent lire, et que les modalités de la compréhension ne sont plus à l'ordre du jour : "ils estiment qu'on sait lire, que le livre c'est acquis donc », "ils estiment qu'on fait ça depuis la primaire que ça doit être acquis, qu'on aurait pas à revenir sur le livre » (Dimitri). La question d'un enseignement explicite des stratégies de compréhension se pose donc ici de façon très nette.

\section{Prémices de passages à la lecture}

Qu'en est-il de leur rapport personnel à la lecture ? Lisent-ils lorsqu'ils n’y sont pas contraints ? Quoi ? Et pour quelles raisons ? Dans ce cadre là éprouvent-ils du plaisir et de l'intérêt à ces lectures buissonnières ? Existent-ils des ponts entre pratiques scolaires et extrascolaires de lecture ? Entre lecture et autres activités langagières ?

Premier constat : à l'instar de «l'écriture extrascolaire des collégiens » (Penloup, 99), la pratique de la lecture n'est pas un terrain en friches. Mais il faut insister, creuser, reformuler la question de différentes manière, tant ces élèves font de la résistance et répugnent à l’idée de se présenter comme lecteurs : «je lis après des magazines quoi, mais c'est pas ce que j'appelle de la lecture, c'est des méthodes, c'est différent, j'appelle pas ça de la lecture » (Rapaëlle).

Pourtant les lectures sont nombreuses, la plupart du temps reliées à un centre d'intérêt privilégié , et font l'objet d'un investissement important: "quand je lis c'est pas pareil, c'est pas une histoire, parce que je lis des trucs de self défense ou l'encyclopédie des arts martiaux, sur ce qui me plaît quoi, mes loisirs », "j’ai plein de livres sur ça », "des fois je sors pas de chez moi, je vais même pas voir mes copains, quand je suis dedans j'en sors plus » (Benoît), "des romans j'en lis pas tellement parce que je sais pas trop mais c'est souvent sur Internet que je lis des textes, des petits textes, des paragraphes qui parlent de l'histoire, sur la guerre, sur les armes, comment étaient les bunkers, comment ils se cachaient, tout ça ça m'intéresse, les différents alliés", (Cyrille), "les livres euh qui expliquent des trucs d'informatique comme ça, j'aime bien, je lis comme ça sans hésiter, j'arrête pas » (Anthony), "Les revues de pêche, chasse, des choses comme ça, c'est des choses pas liées au collège. J'ai lu un gros livre sur la chasse, donc tout ce qui est gibier, ça ça m'intéresse » (Naïm) 
Deuxième constat : Les élèves évoquent la nécessité de relire, de revenir sur certaines lectures qu’ils n’ont pas été capables de lire à un moment donné, qu'ils ont laissées en attente, et sur lesquelles il vont revenir, de leur propre initiative : «je lis Tintin, j’ai toute la collection, j’aime bien, je les ai depuis la $6^{\text {ème }}$, mais je les ai commencés à partir de la quatrième », (Léo)

«J'avais commencé les Dix petits nègres chez moi et ça m'a plu parce que l'année dernière on l'avait déjà étudié et j'ai relu et ça m'a plus » (Benoît).

C’est pourquoi aussi certains évoquent leur choix d'acheter certains livres, pour justement pouvoir y revenir autant que nécessaire : "surtout j’achète aussi, oui comme ça après je peux les relire, si j'ai pas bien compris », "faut prendre son temps, quand on comprend pas faut relire » (Yohan)

Ces commentaires sont intéressants car ils montrent comment s'installent de façon très progressive les habitudes de lecture, comment se constitue peu à peu le "patrimoine » du lecteur en herbes : certaines lectures jugées trop ardues sont consignées, mises en attentes, pour être reprises lorsque le lecteur se sent mieux armé pou les aborder. ${ }^{2}$ Le problème soulevé ici c'est peut-être que l'école n’est guère patiente, et qu'elle demande aux élèves de mordre à l'hameçons de la lecture avant même qu'ils aient toutes leurs dents...

Autre constat : d'autres média, non exclusivement livresques ou textuels, peuvent, dans certaines situations, venir à la rescousse de la lecture. C'est le cas notamment pour les lectures privées (c'est-à-dire sans lien avec les lectures faites dans le cadre scolaire, uniquement motivées par un centre d'intérêt personnel). Parfois, c’est la télévision qui motive la lecture, comme en témoignent les propos de Dimitri :

«j'avais lu euh un livre d'un personnage que j'aimais bien et le livre faisait plus de 500 pages et je l'ai lu et j'ai pu tout retenir », "je l'ai vu à la télé et je l'ai acheté tout de suite », " on est dans son truc quand on connaît bien c'est parlé dans des termes plus familiers quoi plus pour les jeunes, on se lance plus vite dans l'histoire quoi, on aime bien celui qui raconte, on a envie de savoir ce qu'il a fait, ça va plus vite », "j'ai pas vu les pages passer ». Lorsqu'on demande à Dimitri ce qu'il a ressenti à la fin de cette lecture il exprime une certaine fierté, et a l'impression d'avoir franchi un cap : "c'était bien j'avais l'impression d'arriver au même point que les autres », "si les autres livres c'était pareil et si j’arrive à lire comme ça les autres livres en français ce serait bien ».

Mais le support vidéo peut également être utilisée à cette fin dans le cadre scolaire, et cela est perçu de manière très positive par certains élèves, pour qui la télévision est le premier (ou l'un des premiers) vecteur culturel. C'est encore le cas de Dimitri, qui dit, concernant l'étude de Dracula : "tout le monde en a entendu parler à la télé ou quelque part d'autre donc on avait envie de savoir mieux ce qui se passait ».

Même chose pour Cyrille qui évoque lui aussi l'aide apportée par ce support dans le cadre des lectures faites en français :

«Une fois qu'on a vu le film j'ai bien aimé mais sinon j'aimais pas », "le livre que j'ai assez bien compris c'est le voyageur sans bagages, au début j’y comprenais rien et à la page 50 à peu près on a regardé le film et là je suis bien arrivé à lire et je suis rentré dedans, la première chose que j'ai faite après avoir fini mes devoirs le soir c'est finir le livre, alors qu'avant le livre il était au fond du tiroir et ... », " un livre c'est beaucoup différent quand même du film, y a beaucoup plus de choses dans le livre, donc même si on fait des contrôles sur le livre et qu'elle pose des questions un peu difficiles si on n'a pas lu le livre on peut pas répondre même si on a vu le film, mais on pourrait regarder le film avant de lire le livre comme ça on pourrait se faire une idée ", "c'est pour voir l'ambiance, le caractère des personnages, voir comment ils répondent parce qu'on lit mais on met pas le ton si on lit dans notre tête, et après on arriverait à se mettre une petite histoire dans notre tête, à mettre en scène ».

Cyrille voit aussi un lien entre lecture et jeux vidéo : " enfin ça a pas trop à voir avec la lecture mais si quand même, c'est que dans les jeux vidéo juste avant de faire une bataille y a deux ou trois pages qui expliquent comment ça va se dérouler et y a des jeux qui sont assez réels, j'en ai trois, et j'aime bien suivre l'histoire, je fais pas bêtement le jeu comme ça, là je suis bien passionné je sais pas pourquoi mais j’aime bien ».

Entrer en lecture, sous toutes ces formes, implique donc d'avoir du temps, de pouvoir relire, de pouvoir faire le lien entre les lectures faites en classe, ses centres d'intérêt, et les média les plus familiers (en l'occurrence ici la télévision,

\footnotetext{
${ }^{2}$ Voir à ce propos le passionnant travail réalisé par Dominique Dourojeanni sur les jeunes adeptes de « Chair de Poule », et la construction progressive, de leurs habitudes de lecture personnelle.
} 
ou Internet par exemple). L'entrée en lecture implique aussi un certain partage, une invitation qui passe par la parole : s'ils ont franchi le cap ou sont en train de le faire, c'est parce quelqu'un les y a invités verbalement. Ce passeur de lecture est souvent le professeur de français. Mais pas toujours. Ce peut être une personne proche, en qui on a confiance, avec qui on a tissé des liens affectifs :

" y en a un que j'ai bien aimé, c'est ma sœur qui m’avait dit de le lire, Moi Christiane F, 13 ans et demi, droguée, prostituée, celui-là il est bien, parce qu'en fait c'est une biographie, elle raconte son mal être » (Benoît ), "ben en fait je savais pas du tout à quoi m'attendre et j'avais plusieurs amies qui m'en parlaient en me disant que c'était super bien, super bien, super bien, et comme c'est deux amies avec qui je m'entends super bien et qu'on a les mêmes goûts, j'ai dit pourquoi pas je vais essayé, et finalement ouais, je sais pas ce qui m'accroche exactement mais j'aime » (Raphaëlle).

A partir de là le rapport à la lecture peut basculer, l'image de l'élève réfractaire se fissurer, le lecteur devenir plus confiant, laissant présager bien d'autres lectures encore : "par exemple moi je prends l'année dernière, y avait des livre qui m'intéressaient pas du tout, je m'en rappelle plus, et on a lu le livre de Robinson ou la vie sauvage, non vendredi ou la vie sauvage, et y m'a bien plus celui là et je l'ai fini en beaucoup moins de temps que je fais normalement et je pense que c'est à partir de là si on trouve un bon livre... » (Léo).

L’analyse empirique du rapport personnel à la lecture des élèves qui vient d’être présentée montre bien le développement de compétences et d'habitudes de lecture, mais ces « prémisses de passages à la lecture » sont finalement peu relayées ou investies par l'enseignement. Or la lecture n'est pas coupée des autres activités langagières. Jouer, regarder la télé, lire, chercher de l'information sur Internet, chatter, parler, écouter, imaginer, rêver, penser, apprendre, tout se tient ou devrait se tenir. C'est un peu ce que nous suggèrent ici ces élèves...Et s'ils n'avaient pas tout à fait tort ? Mais est-ce le cas dans le cadre des activités pédagogiques, notamment en français, où les textes proposés exigent pourtant des compétences textuelles et des accointances culturelles que sont loin de posséder tous les élèves ? Sans doute pas, et c'est peut-être là que se situe le problème : ces élèves savent lire, mais ne savent pas lire ni apprécier ce qu'on leur propose de lire. Pour eux la lecture littéraire demeure hors de portée, inaccessible, non pas tant par manque de compétences, mais plutôt par manque de "pratiques » permettant de lui donner corps et sens dans le contexte (social, culturel) qui est le leur. Il semble donc que pour ces lecteurs encore fragiles, la lecture, notamment littéraire, ne puisse passer que par une mise en forme (mise en voix, mise en images, en paroles, en gestes, etc...) pour réellement s'incarner dans le quotidien de la classe, et entrer en résonance avec d'autres lectures, d'autres écrits, d'autres paroles, et de façon plus fondamentale, avec l'ensemble des pratiques langagières ${ }^{3}$. Cela nous ramène à la question des outils de socialisation de la lecture et à la problématique non résolue des médiations culturelles.

\section{Lecture et échec scolaire : les ratés de l'école}

La deuxième partie de cette contribution est consacrée aux élèves considérés en échec scolaire soit parce qu’ils n’ont pas intégré un cursus général soit parce qu’ils en sont sortis.

Un consensus s'est construit sur le fait que la maîtrise des compétences langagières et plus particulièrement celles relatives à l'écrit est déterminante dans la réussite scolaire, comme le mettent en évidence à la fois le rapport final de l’INRP sur la production d'écrits et la construction des savoirs dans les disciplines scolaires ${ }^{4}$, et les travaux de l'équipe ESCOL ${ }^{5}$. De là certainement la pression mise sur la lecture, comme on l'a encore vu ces derniers mois avec le faux débat sur les méthodes de lecture, les discours alarmistes, le comptage des illettrés, des faibles lecteurs, des dyslexiques etc. On ne peut bien évidemment pas nier que certains élèves ont des difficultés avec la lecture et qu'il faut les aider « à surmonter leurs ratés de l'apprentissage de la lecture ». Pour autant, tout ne va pas de soi et il paraît nécessaire de continuer à questionner certaines réponses construites trop hâtivement (y compris par les chercheurs) et certaines explications établies aujourd'hui comme des évidences afin de mieux cerner le rapport entre l'échec scolaire et la maîtrise de l'écrit, et plus particulièrement la lecture. Ne pas savoir bien lire conduit-il forcément à l'échec ? Et inversement bien lire conduit-il forcément à la réussite scolaire ? Dans quelle mesure l'école ne construit-elle pas de l'échec langagier et scriptural ? Ainsi osons explorer cette idée que les " ratés de l'apprentissage de la lecture » ne sont peut-être pas forcément les ratés de la lecture, et osons nous demander si ce n'est pas l'école qui rate quelque chose en matière d’apprentissage de la lecture avec certains élèves

3 Pour Bertrand Daunay (2002) la «lecture en réseau » par exemple «ne peut créer un pont entre des pratiques scolaires et non scolaires que si la liberté de lecture des livres du réseau est donnée aux élèves et si la diversité des supports de lectures (livres ou non ; faciles ou difficiles, longs ou courts, littéraires ou non, etc.) permet un ancrage dans les pratiques non scolaires de lecture ». (p.68).

${ }^{4}$ INRP, Production d'écrits et construction des savoirs dans les différentes disciplines scolaires : gestion de l'hétérogénéité des élèves, décembre1999

${ }^{5}$ CHARLOT B., BAUTIER, E., ROCHEX, .Y., (1992), Ecole et savoir dans les banlieues ... et ailleurs, Paris, Armand Colin 
Cette partie vise donc à construire un contre point en regardant la question de l'apprentissage et de la pratique de la lecture selon deux prismes construits à partir de deux groupes d'élèves

- d'une manière rétrospective à partir du point de vue des lycéens décrocheurs,

- d'une manière décalée à partir du point de vue des collégiens de segpa qui eux n’ont pas réussi à intégrer le cursus secondaire général.

Ces élèves ont en commun bien qu'en échec scolaire de s’être accrochés. Les élèves décrocheurs que nous avons rencontrés au CLEPT après être sortis volontairement du système scolaire, parce qu'ils s'y trouvaient en difficulté y retournent avec le projet scolaire d'obtenir le baccalauréat). Ils sont motivés, ou remotivés, et se réengagent dans une dynamique d'apprentissage qui devrait les conduire à, comme l'indique le titre de ce numéro, « surmonter les ratés de l'apprentissage ». Quant aux élèves de $3^{\circ}$ segpa, bien que déclarés non aptes à intégrer le collège, ils n'ont pas quitté le système scolaire et ne sont pas en rupture de ban avec lui. Ils ont suivi un parcours complet et très cadré, mais décalé par rapport au cursus général. A la fin du parcours, leur formation est sanctionnée par un examen, qu'ils souhaitent réussi et pour lequel ils se mobilisent.

Pourquoi ces élèves intéressent-ils la recherche en lecture ? Tout d'abord parce que leur profil exclut de recourir au motif de la démotivation pour expliquer l'échec. Ensuite, parce que leur âge et leur histoire scolaire les situant en fin de parcours, il est possible d'appréhender avec eux les problèmes de manière rétrospective. Ces élèves peuvent parler de l'échec, et du rôle de l'écrit avec un certain recul. Ils peuvent aussi en revenant sur leur parcours scolaire identifier quels éléments ont pu constituer des obstacles à l'apprentissage (événements particuliers, modalités scolaires, types d'évaluation, etc.). Enfin, ces élèves ayant un certain âge, certains parmi eux font preuve d'une maturité intellectuelle et psychologique suffisante pour expliquer et expliciter ce qui a provoqué l’échec, et quel rôle y a joué leurs difficultés en lecture - écriture.

Nous avons donc mené avec ces élèves du Clept ( Collège - Lycée Elitaire pour Tous) de Grenoble et de la $3^{\circ}$ segpa du collège de La Mure des entretiens sur leur parcours scolaire avec l'objectif de rechercher qu'elle pouvait être la place de l'écrit dans l'échec scolaire auquel ils ont été confrontés. Les thèmes abordés lors de ces entretiens portent sur les points suivants :

- $\quad$ perception des activités de lecture et écriture en classe, dans les différentes disciplines et pas seulement en français : nature de ces activités, modalités, fonctions,

- $\quad$ intérêt pour ces activités

- $\quad$ place et rôle de ces activités dans les processus d'apprentissage dans les différentes disciplines.

- $\quad$ réussites et difficultés avec l’écrit

- $\quad$ pratiques de lecture et d'écriture extrascolaires

Nous choisissons ici de rendre compte des propos et avis de trois élèves, Mathias, Laurine et Marion, que nous considérons comme représentatifs de profils d'élèves pour lesquels le lien qui s'est tissé entre lecture et échec scolaire questionne les pratiques pédagogiques. On peut considérer que ces élèves constituent des exceptions : nous ne le croyons pas, dans la mesure où nous avons retrouvé dans les propos de leurs camarades des questionnements similaires. Par ailleurs, mieux comprendre le rapport entre échec scolaire et lecture passe par la voie de la centration sur le sujet élève et lecteur pris dans son individualité et sa singularité et sur ce qu'il construit et comprend de son activité de lecteur et de son apprentissage de la lecture (Charlot, alii 1992). Enfin, l'enseignant travaille avec des individus et pas avec des groupes sociaux. Certes ce qu'il sait des pratiques socioculturelles l'aide à lire et décoder les pratiques individuelles, mais il a surtout à " décoder » des individus pour leur proposer des démarches d'apprentissage adaptées.

Les réponses que nous avons obtenues lors des entretiens nous montrent qu'en matière d'apprentissage de la lecture et de recherche sur ce qui le favorise ou y fait obstacle il pourrait être profitable de découpler des paradigmes considérés jusque là comme fortement liés, à savoir la lecture et l'écriture, la pratique de lecture et la réussite scolaire, la lecture scolaire et la lecture extra scolaire. Ces analyses se présentent davantage comme des hypothèses pour affiner la réflexion sur la lecture.

\section{Rapport à l'écrit, lecture et écriture}

Les discours des élèves engagent, dans le vaste champ de l'acculturation à l'écrit, à distinguer lire et écrire pour tenter de mieux formaliser comment le sujet en construit la spécificité. Certes lire et écrire sont deux compétences et pratiques scripturales et l'école s'efforce de les mettre en rapport l'une avec l'autre afin d'en articuler les apprentissages, cependant elles demandent de la part des sujets des habiletés et des investissements totalement différents. Il apparaît que pour certains apprenants le rapport à l'écrit se construit plus difficilement quand il s’agit de lire que quand il s'agit d'écrire. 
C'est ce que suggèrent les propos de Mathias, élève de $3^{\circ}$ de segpa, qui annonce d'emblée qu'il n'aime pas l'école, qu'il a des difficultés en lecture et écriture - ce qu’a confirmé son professeur, qu'il n’aime pas lire et qu'il ne lit pas, sauf les manuels d'entretien des machines agricoles et le livret du code de la route (voir ci-dessous). Le portrait que Mathias construit de lui-même est donc celui de l'élève en échec avec l'écrit, donc avec l'école. Pour autant, Mathias affirme qu'il n'est pas « dérangé » par l’écriture.

E : Je reviens au début pour toi l'école c'est lire et écrire - $M$ : Oui - E : Et ça tu n’aimes pas faire lire et écrire ce n'est pas ton truc - $M$ : Non ce n'est pas mon truc - [...]-E: Et pour écrire alors qu'est-ce qui te déplaît - $M$ : Ecrire ça ne me dérange pas - E: Ecrire ça ne te dérange pas oui pourquoi - $M$ : Non ça ne me dérange pas d'écrire - E: Mais est-ce que tu aimes bien ou est-ce que c'est simplement que ça ne te dérange pas - $M$ : Non ça ne me dérange pas du tout - E : D'accord et qu'est-ce que tu écris alors qu'est-ce que tu es amené à écrire- $M: B e n$ pour répondre aux questions - E : Oui tu es obligé ou sinon quand tu es chez toi que tu n'es pas à l'école est-ce que tu es amené à écrire des fois ou pas - $M$ : Non rarement non

Parallèlement, Mathias affirme qu'il lit quand ça l’ « arrange »

$E$ : Et tu lis des magazines des fois ou des revues - $M$ : Non je lis quand ça m'arrange - E : Tu lis quand ça t'arrange alors pour quoi par exemple - $M$ : Ben je ne sais pas des fois quand mon père il achète quelque chose le livre -d'entretien des fois je le prends et je fais xxx je regarde des trucs comme ça-E : Tu lis des modes d'emplois ou des trucs comme ça-M:Oui

Une fois remarqué, selon une analyse plusieurs fois formulée déjà, que ces pratiques de lecture et écriture sont liées à des situations quotidiennes et circonscrites à une utilité précise, ce qui au bout du compte les rend peu fréquentes, il n’en reste pas moins que Mathias envisage lecture et écriture de manière presque antinomique, comme le suggère l'emploi du couple arranger / déranger, construit à partir du mot " ranger » . Comment entendre ce que dit Mathias ? Le terme de "déranger » suggère que pour cet élève écrire ne provoque pas de "désordre » en lui, voire qu'écrire produit le contraire du désordre, alors que lire « dérange ». En effet Mathias dit qu'il lit quand « ça l'arrange », c'est-à-dire quand ça lui est utile et que cela lui permet de mettre de l'ordre dans ce qu'il doit savoir, en particulier pour faire fonctionner les machines agricoles. Ainsi on peut faire l'hypothèse que écrire relevant d'une expression personnelle l'individu en contrôle en partie le processus et peut construire en quelque sorte son propre ordre. En revanche la lecture requiert une décentration de l'individu par rapport à lui même pour prendre en compte l'autre qui écrit ; il faut entrer dans sa logique, dans ses mots et son discours, c'est-à-dire entrer dans un autre ordre. Dans chacune de ces pratiques, le rapport à l'écrit qui se construit est différent et peut prendre pour l'individu des valeurs antinomiques. Ces aspects de la lecture, de sa pratique et de son apprentissage ne trouvent pas toujours place dans des démarches pédagogiques qui privilégient l'articulation décodage / compréhension ${ }^{6}$.

\section{Lire et réussir à l'école}

Maîtrise de l'écrit et réussite scolaire sont fortement liés. Il est indéniable que, les différents apprentissages disciplinaires prenant appui sur l'écrit, l'élève en difficulté avec l'écrit risque d'être en difficulté scolaire. Pour autant construire le rapport maîtrise de l'écrit / réussite scolaire principalement dans ce sens ne permet pas d'en explorer toutes les implications pédagogiques. On peut en effet bien savoir lire et rater à l'école. Parmi les élèves rencontrés, deux ont réussi leur apprentissage de la lecture, et ont suivi une scolarité primaire satisfaisante, puis elles ont connu l'échec au collège. Quel enseignement tiré de cette situation dans laquelle se trouve un certain nombre d'élèves.

\section{Laurine : Aimer lire et rater à l'école}

Laurine a suivi un parcours en primaire plutôt satisfaisant, mais n'a pas franchi les obstacles du collège. Elle se retrouve donc orientée en segpa où au bout du compte elle réussit pas mal puisqu'elle obtient en $3^{\circ}$ une moyenne générale de 18 . Son professeur pense qu'elle pourra intégrer une classe de BEP sans problème et qu'elle y réussira. Ces succès scolaire ne doivent pourtant pas faire oublier que Laurine n’a pas trouvé sa place dans le cursus général et qu’elle a été déclarée en échec. Or son

\footnotetext{
${ }^{6}$ GUERNIER, M.C., (2000), « Je veux leur faire comprendre que quand on lit il faut comprendre. Discours ordinaires d'enseignant sur la lecture », Les Cahiers du Français contemporain $n^{\circ} 7$ : " Lire pour comprendre : pratiques de lecture et fractures du sens » (dir. Michel Dabène)
} 
profil d'élève et de lectrice contredisent la dialectique rapport au savoir / rapport à l'écrit / échec / réussite. En effet Laurine est une élève motivée, qui aime l’école, parce qu'elle aime apprendre et réfléchir comme elle le dit elle-même.

E : Est-ce que toi tu aimes bien l'école ou pas ou est-ce que ça t'ennuie - L : Non j'aime bien l'école [...] ben j'aime bien apprendre des choses ben j'aime bien les profs aussi ils sont sympa et puis je ne sais pas [... ] ben apprendre des nouvelles des nouveaux trucs des nouvelles méthodes - [...] - E : D’accord alors par exemple qu'est-ce que tu aimes bien faire en maths - $L$ : Ben des problèmes euh des opérations j'aime bien un petit peu de tout en maths [...] ben il fallait réfléchir et il fallait savoir ce qu'il fallait faire [...] j'aime bien ben j'aime bien un peu toutes les matières

Ainsi Laurine envisage les activités scolaires dans leur dimension intellectuelle : " apprendre », "réfléchir ». Il ne s’agit pas seulement d'accomplir des tâches ou d'exercices, mais bien d'activer une compétence intellectuelle. Par ailleurs, Laurine a une pratiques d'écriture personnelle : elle écrit chez elle dans un cahier des histoires, des poèmes, des chansons. Enfin, Laurine aime lire, lit beaucoup et présente toutes les caractéristiques de la bonne lectrice : appétence, autonomie : elle fréquente la bibliothèque municipale et le cdi du collège, compétence : elle comprend, connaît les livres et les auteurs, milieu porteur : elle évolue dans une famille lectrice où on échange sur les livres, investissement sur l'écrit : elle préfère le livre au film.

$E:$ Tu aimes bien lire - L : Oui - E : Qu'est-ce que tu lis alors par exemple - L : Je lis Chair de Poule des fois je lis des trucs d'action enfin des livres d'action - $E$ : Où tu les trouves ces livres - $L$ : Ben soit au CDI d'ici à la bibliothèque de la Mure ou soit chez moi - E : D'accord tu en empruntes beaucoup - L : Ben j'en emprunte deux à chaque fois et puis après une semaine je les rends et ainsi de suite - $E:$ Tu lis beaucoup alors- $L:$ Ben des soirs je lis avant de m'endormir je lis toujours un quart d'heure une demie heure - E : Et à la bibliothèque de la Mure tu prends des livres aussi - L : C'est ma mère et mon frère ils prennent et après moi je les lis - E: D'accord donc ce n'est pas toi qui vas les choisir à la bibliothèque - $L$ : Si des fois ma mère elle me donne sa carte - $E$ : D'accord alors qu'est-ce que tu lis par exemple tu $m$ 'as dit des Chair de Poule et puis quoi d'autre encore - $L$ : Des livres d'action - E : Qu'est-ce que tu appelles des livres d'action tu peux me donner des exemples - $L$ : Comme dans Stephen King par exemple ou dans Harry Potter il y a de l'action - E : Tu les as tous lus les Harry Potter parce que c'est gros quand même - $L$ : Ben là je suis au $5^{\circ}$ mon frère est en train de lire le $6^{\circ}-E$ : Ah d'accord et vous en parlez après avec ton frère ou pas - $L$ : Après oui on en parle et puis après ma mère elle achète les DVD et on les regarde - $E$ : Ca te plaît aussi les films - $L$ : Oui mais des fois ils enlèvent des trucs des livres quoi enfin moi j'aime bien lire le livre et puis après regarder le film - E : D'accord et tu es déçue par le film ou pas - $L$ : Non mais c'est parce que des fois ils enlèvent des morceaux parce qu'il est gros - E : Et donc en classe ce que vous lisez qu'est-ce que vous lisez en classe - $L$ : Ben on lit des exercices et puis des fois quand on a fini nos exercices on a le droit de prendre un livre - E : D'accord alors les exercices tu trouves ça intéressant comme lecture ou pas - L : Pas trop en fait je prèfère les livres comme Harry Potter ou - E : Et ça ça t’intéresse les livres qu'il y a en classe ou pas - L : Oui je prends de temps en temps quand j'ai fini avant les autres [...] - E : D'accord et toi tu penses que tu es une bonne lectrice - $L$ : Oui ça va- E: Qu'est-ce qui te permet de dire que tu es une bonne lectrice - $L:$ Ben je lis ....je comprends ce que je lis et je lis assez vite

Les propos de Laurine sont explicites et dessine donc un profil de bonne lectrice. Mis en rapport avec l'échec scolaire de Laurine, ils suggèrent une question cruciale : pourquoi cette compétence n'a-t-elle pas trouvé à être investie à l'école et dans un processus de réussite scolaire ? Doit-on considérer qu'il y a là un raté et qu'il incombe à l'école ? Les propos de Laurine et l'enquête en cours n'apportent pas directement la réponse, mais on voit la nécessité de ne pas poser la question du rapport entre la maîtrise de l'écrit et l'échec scolaire seulement de manière inductive sous la forme « ne pas maîtriser l'écrit entraîne l'échec », mais aussi de manière plus problématique.

Marion : décrocher et se mettre à lire

L'exemple de Marion construit une autre dialectique entre maîtrise de l'écrit et échec scolaire, qui paraît encore plus problématique. Marion est aujourd'hui élève en $1^{\circ} \mathrm{L}$ au clept. Auparavant elle a suivi une scolarité normale au collège, a intégé le lycée général et là a décroché. Elle explique ce décrochage par la pression qu'exerçaient sur elle les manières scolaires.

E : Est-ce que la place de l'écrit a eu un rôle dans le fait que tu as eu des problèmes à l'école dans le système traditionnel M : Oui [...] enfin moi c'était aussi vachement lié avec l'apprentissage aussi parce que vu que justement j'étais tout le temps sur les feuilles de pompes normal qu'après pour écrire c'est très dur [...] parce que tu stresses déjà que dans ta tête c'est vide étant donné que c'est tout écrit sur ton bout de papier donc après c'est sûr que pour écrire il faut que tu inventes je peux dire c'est simple si je fais du blabla c'est simple d'écrire et après si tu veux faire quelque chose de correct c'était pour moi 
très dur oui [...] oui moi ça me soulait la mise à l'écrit je rentrais chez moi je n'avais pas envie d'écrire des trucs quoi [...] je rentrais je n'avais qu'une seule envie c'était de poser mon sac d'écouter de la musique de faire autre chose tout sauf du scolaire quoi - E : Et à cette époque là est-ce que tu écrivais pour toi toute seule comme tu le fais maintenant - M : Non non pas du tout [...] je faisais vachement de sport vachement de dessin mais l'écriture non

Dans ce contexte scolaire que Marion décrit comme stressant, la pratique de l'écrit perd son sens et paradoxalement, il faut quitter l'école pour renouer avec l'écrit et la lecture.

E : Et la lecture pareil - M : Et la lecture non c'est au moment où j’ai décroché que j'ai commencé à lire [...] vu que les cours ne me plaisaient pas il y a quand même un manque les cours c'est quand même quelque chose qui te rentre dans la tête enfin et là une envie de me nourrir de littérature de phrases enfin de quelque chose et là ça m'a fait vachement de bien de pouvoir rester chez moi et de passer des journées à lire là par contre mes bouquins je les dévorais assez vite [...] alors j'ai lu le voyage en Iran de Betty Mahmoody ça me plaisait parce que c'était une histoire vraie et en même temps je voyageais dans le pays et cela me faisait apprendre la culture de ce pays bon après ce n'était peut-être pas objectif je ne peux pas juger [...] j'avais essayé de lire le Voyage de Gandhi mais là je me suis dit reprends ton histoire un peu avant de vouloir [...] après il y a plein d'autres trucs Les racines des palétuviers ça c'est un médecin qui est allé en Inde qui s'est installé et qui a réussi à monter un truc -E: Tu n'allais pas en cours là donc tu étais chez toi tu occupais tes journées à lire - M : Ah oui et puis à réfléchir vachement à tout ce qui c'était passé justement au niveau scolaire [...] je pataugeais je ne suis pas allée en cours [...] ça a duré un an $-E$ : Et là tu as beaucoup lu $-M$ : Oui [...] j'avais d'autres idées qui naissaient et que je n'avais pas forcément en cours

Dans ces propos de Marion, on voit que la lecture se présente comme une nourriture qui permet à l'individu de se structurer et de se construire. Ce qui interroge ici c'est que ce travail se fait non pas seulement en dehors de l'école, mais surtout contre l'école.

Ainsi il apparaît que pour mieux explorer l'articulation entre ces deux paradigmes « savoir lire » et « réussir à l'école», il est certainement nécessaire de les découpler. D’une part pour poser la question : d’où vient l'échec scolaire ? Qu'est-ce qui le provoque ? Afin de mettre de côté, comme le proposent l'équipe d’Escol, les réponses strictement sociologisantes ( écart culturel) et psychologisantes (manque de motivation). En effet, ni Laurine ni Marion ne manquent de motivation, ni elles ne sont en difficulté avec l'écrit. Or pour l'une cette maîtrise ne parvient pas à se concrétiser en réussite scolaire et pour l'autre l'épanouissement lectural non seulement ne peut se réaliser que lorsque l'école s'arrête mais aussi permet en partie de résoudre les difficultés créées par l'école elle-même. Ainsi la dialectique pratique de lecture / réussite scolaire semble ici particulièrement complexe. Les réponses sont à chercher en partie dans les pratiques pédagogiques qui concernent la lecture et en mettant en rapport ce qui est trouvé dans les discours des élèves et l'analyse des pratiques de classe. Ce que nous n'avons pas ici. Mais ce que montrent les entretiens avec les élèves lecteurs et en échec c'est que le rapport maîtrise de l'écrit / échec scolaire doit être mieux ausculté et que les pratiques pédagogiques, pas seulement liées à la lecture d'ailleurs, doivent être interrogées.

Lecture scolaire / lecture extra scolaire

On le sait l'école propose et promeut des manières de lire spécifiques, que l'on a qualifié de lecture analytique ou objectivante, et qui s'opèrent sur des supports spécifiques, plus particulièrement des textes littéraires, qui ont un intérêt didactique ( Viala ${ }^{7}, 1995$ et Guernier $\left.^{8}, 1999\right)$. Ce faisant l'école a construit une conception de la lecture liée à son ancrage « lettré » dans le système scolaire : la lecture est le domaine des professeurs de français ou de la discipline français. Cette conception est également liée aux représentations sociales et culturelles récentes qui dans le courant du $20^{\circ}$ siècle font passer la lecture dans la sphère du culturel. Or ces pratiques et conceptions scolaires sont en décalage avec les pratiques de certains élèves.

Ne pas savoir lire ce que propose l'école - Le raté des supports

\footnotetext{
${ }^{7}$ VIALA, A., (1995), “ Demandez le programme ”, in Seibel, B., (dir.), Lire, faire lire. Des usages de l'écrit aux politiques de lecture, Les Editions Le Monde, pp.319-333

${ }^{8}$ GUERNIER, M.C., (1999), « Lire et répondre à des questions au cycle 3 », Repères, $\mathrm{n}^{\circ} 19$, « Comprendre et interpréter les textes à l’école », (dir. F. Grossmann et C. Tauveron), Paris, INRP
} 
Revenons au discours de Mathias sur les pratiques scolaires de lecture. Selon la typologie de l'équipe Escol Mathias a construit un rapport pragmatique au savoir. Ce qui est appris doit servir dans la vie quotidienne et en particulier celle de la ferme de laquelle Mathias est issue et où il travaille quotidiennement et où il travaillera définitivement et à temps complet à sa sortie du collège. Cette activité lui plaît et lui apporte beaucoup de satisfactions. A la différence de l'école dont il dit qu'il se passerait si c'était possible. L'école n’intéresse pas Mathias, non pas parce qu'il n’y apprend rien, mais parce qu'elle lui propose des « choses » qui ne l’intéressent pas, y compris en matière de lecture.

E : Et ça tu n'aimes pas faire lire et écrire ce n'est pas ton truc-M : Non ce n'est pas mon truc- E : Et quand tu es chez toi tu lis quoi par exemple - $M$ : Je fais le permis 125 donc j'apprends les panneaux et tout ça - E : Et tu aimes bien faire ça$M:$ Oui - E : Et quand tu apprends ça dans le livret cela te paraît difficile ou facile - $M$ : Ben là ça va non ce n'est pas top dur - E : D'accord et là du coup lire ça ça t'intéresse - $M$ : Oui - E : Est-ce que tu lis autre chose des fois chez toi - $M$ : Non pas souvent - E : Tu lis le journal -M : Non - E : Et tu lis des magazines des fois ou des revues - M : Non je lis quand ça m'arrange - E : Tu lis quand ça t'arrange alors pour quoi par exemple - $M$ : Ben des fois quand mon père il achète quelque chose le livre d'entretien des fois je le prends et je regarde des trucs comme ça- E : Et sur les machines agricoles tu lis des choses ou pas - M: Je lis les livres d'entretien - E : Mais c'est compliqué les livres d'entretien non - M : Non je les lis doucement j'ai le temps le soir [...]des fois les boutons je ne sais pas à quoi ça sert alors des fois ça me ça peut toujours servir [...] - E : Donc ce n'est pas du tout la même chose que ce que vous lisez à l'école - M : Ce n'est pas pareil $E$ : Et si madame L. vous faisait lire des livres d'entretien ça t'intéresserait l'école ou pas - M : Oui - E : Tu viendrais un matin à l'école et on vous ferait lire le livre d'entretien d'une machine tu trouverais que c'est étrange ou normal - M : Etrange parce que ce n'est pas pareil [....] on apprend surtout ce qu'on sait ou des trucs comme ça quoi - E : Et qu'est-ce qu'elle vous fait lire madame L. - M : Ben maintenant c'est le CFG donc des textes et on doit répondre aux questions des trucs comme ça - E : Et ces textes là tu te souviens de quoi ils parlent - $M:$ Non - E : Et répondre aux questions tu arrives bien ou pas trop -

$M:$ Des moments oui des moments non [...] mais on les trouve toujours - E : D’accord et à ton avis poruquoi en $3^{\circ}$ on vous fait faire ce genre de travail avec les textes - M : Ben pour le CFG pour avoir l'examen- E : Donc toi tu te dis on peut supprimer l'école on n'en a pas besoin est-ce que tu irais jusqu'à dire ça - M : Si quand même si je n'avais pas les cours je ne saurais pas trop bien lire et des choses comme ça je ne saurais rien

On le voit les livres qui intéressent Mathias sont le livret du code de la route et les manuels d'entretien des machines agricoles. Or, l'école lui propose des textes à partir desquels il faut répondre à des questions (Guernier, repères) et qui servent essentiellement pour obtenir un examen. Dans cette perspective ces textes ont un intérêt et un ancrage strictement scolaires à la différence des livres en rapport avec une activité et une situation de la vie professionnelle. Mathias admet que ce travail sur les textes est utile pour avoir le CFG et pour aller en apprentissage, cependant cela ne suffit pas à les rendre intéressants. Pour lui l'utilité scolaire ne fonctionne pas comme un motif suffisant. En même temps, Mathias considère que l'école est utile et qu'elle lui apporte quelque chose. Ce qui lui pose problème c'est le décalage. Ainsi Mathias est d'accord pour apprendre, mais des choses qui le concernent. Si on lui faisait lire des manuels d'entretien des machines agricoles, alors il serait intéressé.

L'avis de Mathias, et des élèves qui pensent comme lui, induit forcément une interrogation : pourquoi l'école n’utilise-t-elle pas ces supports qui intéressent les élèves ? D’autant que ces livres ont autant d'intérêt linguistique que les autres. Ne peut-on pas considérer qu'il y a là un raté de l'école quand elle réduit la lecture à un type de texte et une modalité de lecture ? On peut conclure que l’école crée le non investissement sur la lecture scolaire.

\section{Quand l'école empêche de lire}

En dépit du support dont l'intérêt ne rencontre pas toujours l'adhésion de l'élève lecteur, il arrive aussi que se construise ce paradoxe que la prescription scolaire de lecture aboutisse à la non lecture, comme le montrent les propos de Marion.

En effet, l'école prescrit des lectures considérées comme faisant partie de la formation. Le plus souvent ces lectures sont en rapport avec l'enseignement du français, mais pas seulement. Par exemple dans le cadre de sa participation à un atelier photographique Marion doit lire un ouvrage sur un photographe. Ces lectures devant se faire à la maison, l'élève est renvoyé à une pratique personnelle et solitaire de la lecture, non accompagnée qui n’est pas sans lui poser de problème, même quand le lecteur est capable d'une lecture longue et difficile, comme c'est le cas de Marion.

E : Est-ce que dans votre formation au clept vous avez beaucoup de moments où vous lisez pour lire - M : Ben des bouquins en français qu'on doit lire mais ça ça se fait à la maison donc ça après c'est personnel bon moi j'arrive mais je ne dévore pas le bouquin en deux soirs [...] madame A. nous impose des livres elle nous en conseille aussi plusieurs ensuite dans l'atelier on a un bouquin aussi à lire sur un photographe - E : Il faut que vous lisiez Candide en entier vous avez beaucoup 
de temps pour lire Candide - M : Oui voilà bon généralement deux à trois semaines oui [...] ça me paraît beaucoup de temps si je respectais la chose [...] mais moi c'est-à-dire je sais qu'il faut que je lise ce bouquin j'ai un délai de 10 jours donc moi je le lirai dès que j'y penserai enfin 45 jours avant la date fatidique [...] parce que j'ai plein d'autres choses à faire à côté et que dès que je rentre chez moi j'essaie de bosser au maximum le reste [...] c'est vrai que je ne prends pas le temps de lire parce que c'est vrai que tu peux toujours trouver un moment pour lire - E : Et le bouquin sur le photographe tu as fait pareil ou c'est parce que c'est Candide qui est un peu - M: Non le bouquin sur le photographe c'est quelque chose qui m'intéressait déjà plus plus simple à lire c'est-à-dire tu t'arrêtes à une page bon tu peux reprendre facilement sans avoir à faire tout le cheminement - E : Et en plus des bouquins qu'on vous conseille ici ou que madame A. vous impose tu lis comme ça chez toi ou ce n'est pas une activité qui fait partie de tes habitudes - $M$ : Ce n'est pas une des activités la plus développée mais de toute façon pendant les 2 ans qu'il me reste à tenir on en a un peu parlé avec madame A. on n'aura pas le temps de lire enfin à part si je dévore la chose ce qui n'est pas mon cas on n'aura pas le temps de lire et Candide et quelque chose qui me plaît à côté là je vais rentrer en L il faut que je me mette à fond dans tout ce que je vais avoir pour le Bac enfin je veux dire elle nous donne par exemple 3 bouquins à lire mais il y en a 5 à côté qu'il faudrait lire aussi pour pouvoir avoir une plus grande ouverture - E : Et là tu te dis que ça va se passer comment alors - M: Je ne crains pas parce que moi si je suis en $L$ c'est parce que je désire lire et justement apprendre à lire entre guillemets m'enrichir de tout ça [...] mais là ça fait longtemps que je n'ai pas lu un bouquin pour moi

On retrouve dans les propos de Marion ce qui est apparu dans l'entretien de Mathias : la question de l'intérêt pour ce qu'il y a à lire. On ne peut donc pas considérer que ce point n’est pas névralgique. Mais pour Marion cette lecture, même peu intéressée est possible parce qu'elle est motivée par le projet scolaire. En revanche, Marion décrit aussi explicitement le paradoxe de la lecture scolaire, même pour les classes de littéraires et qui consiste à devoir lire sans avoir les moyens de mettre en œuvre cette lecture nécessaire. Par ailleurs, dans cet entretien, le paradoxe est en quelque sorte poussé à son paroxysme dans la mesure où tous les acteurs du système scolaire, y compris les professeurs, l'ont intégré. On n'en vient donc à affirmer que la caractéristique de la lecture scolaire, est qu'elle est à la fois nécessaire et impossible. Il est difficile de ne pas se demander comment les élèves peuvent intégrer cette dialectique et on voit la nécessité d'en mesurer les effets contreproductifs sur les représentations de la lecture.

\section{Conclusion générale}

L’analyse des propos de nos témoins, qu'il s’agisse de collégiens « ordinaires », d'élèves raccrocheurs ou en SEGPA, interroge les pratiques pédagogiques. En effet, tous ces élèves savent lire, mais ne savent pas lire scolairement, c'est-à-dire ne savent pas lire ce que l'école leur demande de lire. Autrement dit, le modèle de lecture proposé par l'école les conduit soit à l'échec, soit à quitter l'enseignement général (c'est le cas de 5 des 6 élèves collégiens « ordinaires » dont il question dans la première partie de ce texte).

Ce que nous renvoient les élèves qui reviennent sur leur parcours c'est d'abord l'inadéquation entre leurs intérêts / désirs et ce qui leur est proposé, entre leurs manières de faire et les manière de faire à l'école. Le principal raté ici semble donc concerner l'incapacité de l'école à prendre en compte l'individu, en travaillant par exemple davantage le lien lecture scolaire/lecture extrascolaire.

Or nous savons bien sait à quel point la reconnaissance des pratiques individuelles des élèves peut constituer l'amorce d'un habitus, en permettant notamment au sujet de se placer dans une « communauté de lecteurs ", incarnée « dans des gestes, des espaces, des habitudes ». C'est à ce prix que « la lecture pourrait alors (re)devenir le lieu d'un échange, du " partage social d'un message » (Privat, 1993).

D’où pour finir la mise à jour d'un paradoxe : l'école, premier prescripteur de lecture, premier lieu d'apprentissage, apparaît en même temps le lieu qui empêche en quelque sorte de pratiquer la lecture...

\section{Références Bibliographiques}

Barré de Miniac, ch. (2000) : Le rapport à l'écriture, aspects théoriques et didactiques, Paris, Septentrion.

Charlot, B., Bautier, E., Rochex, Y., (1992), Ecole et savoir dans les banlieues ... et ailleurs, Paris, Armand Colin

Chartier, A : « La littérature en classe de quatrième, relire pour relier », in Frier, C : Passeurs de lecture, lire ensemble à la maison et à l'école, Paris, Retz, Forum Education Culture.

Daunay, B. (2002) : « Les difficultés de lecture au collège : quelques interrogations », in Recherches n 36, p.51-73.

Delamotte, R. Gippet, F. Jorro, A., Penloup, M. C. (2000) : Passages à l'écriture, un défi pour les apprenants et pour les formateurs, Paris, PUF.

Dourojeanni, D. (2006) : «l'effroi en partage : lecteurs de Chair de poule et échanges épistolaires », in $\operatorname{Argos} N^{\circ}$ ?

Frier, C : Passeurs de lecture, lire ensemble à la maison et à l'école, Paris, Retz, Forum Education Culture. 
INRP, (1999) : Production d'écrits et construction des savoirs dans les différentes disciplines scolaires : gestion de l’hétérogénéité des élèves.

Guernier, M.C., (1999), « Lire et répondre à des questions au cycle 3 », Repères, ${ }^{\circ}{ }^{19}$, « Comprendre et interpréter les textes à l'école », (dir. F. Grossmann et C. Tauveron), Paris, INRP

Guernier, M.C., (2000), « Je veux leur faire comprendre que quand on lit il faut comprendre. Discours ordinaires d’enseignant sur la lecture », Les Cahiers du Français contemporain $n^{\circ} 7$ : « Lire pour comprendre : pratiques de lecture et fractures du sens » (dir. Michel Dabène)

Penloup, M. C. (1999) : L'écriture extrascolaire des collégiens, des constats aux perspectives didactiques, Paris, ESF.

Privat, J. M, (1993) : « l’institution des lecteurs, in Pratiques, n 80, pp.7-34.

Viala, A., (1995), “ Demandez le programme ”, in Seibel, B., (dir.), Lire, faire lire. Des usages de l'écrit aux politiques de lecture, Les Editions Le Monde, pp.319-333

Résumé

L'évaluation de la maîtrise de la lecture est un problème complexe et relève de paradigmes divers : cognition, acculturation, psychologie et didactique. Pour mieux comprendre comment la compétence lecturale se construit, le chercheur doit donc s'intéresser au sujet lecteur lui-même, à son activité et à ce qu'il y investit intellectuellement, psychiquement, socialement et culturellement. Dans cette perspective, nous sommes loin d'avoir investi toutes les pistes.

Partant du constat que, même si les collégiens et lycéens manifestent un certain nombre de savoir faire (lire à voix haute de façon relativement fluide, être capables de répondre à des questions portant sur un texte), leur compétence lecturale est encore largement inachevée, le présent article explore la question de l'apprentissage continué de la lecture dans l'enseignement secondaire : apprend-on encore à lire au collège et au lycée ? Quoi et de quelle façon ? Quels problèmes de compréhension et de pratiques de lecture la scolarité élémentaire a-t-elle résolus et laissés en suspens ? Les élèves sont ils suffisamment outillés pour affronter les textes, notamment littéraires, qui leur sont proposés au cycle secondaire ? Dans quelle mesure les pratiques pédagogiques mises en œuvre peuvent-elles favoriser ou au contraire gêner la continuation des apprentissages dans ce domaine ?

L'article rend compte des réponses que des collégiens et lycéens ont apporté à ces questions dans le cadre d'entretiens semi directifs et vise à décrire et analyser le rapport à la lecture de ces élèves, à mieux comprendre certaines de leurs difficultés et leur manque d’appétence vis-à-vis de la lecture.

Mots clés : Rapport à la lecture / enseignement secondaire / pratiques de lecture scolaire / accompagnement didactique en lecture 